\title{
A test of two different revelation effects using forced-choice recognition
}

\author{
JenNifer C. Major and William E. Hockley \\ Wilfrid Laurier University, Waterloo, Ontario, Canada
}

\begin{abstract}
The revelation effect refers to the finding of an increased propensity to classify recognition test probes as old when they are preceded by a problem solving task. Recent research indicates that revelation effects are dissociable based on whether the revelation task involves an item that is the same as or different than the subsequently presented recognition probe. Using a two-alternative forced-choice design, we found a revelation effect for both words (Experiment 1) and nonwords (Experiment 2) in the condition where the revealed item was the same as the target item (same revelation condition), but no effect when the revealed item was different than either test alternative (different revelation condition). These results were replicated using a mixed list design containing both words and nonwords (Experiment 3). Results support Verde and Rotello's (2004) two-factor account of the revelation effect, which proposes that changes in memory sensitivity underlie revelation effects in the same revelation condition, and that changes in the decision criterion are responsible in the different revelation condition.
\end{abstract}

The revelation effect refers to the phenomenon whereby subjects are more likely to classify studied and unstudied recognition probes as old when they are preceded by a problem solving task compared to when they are not. Since the revelation effect was first demonstrated by Peynircioğlu and Watkins (1988), researchers have demonstrated that a wide range of tasks produce this phenomenon. For instance, the effect has been found when subjects are presented recognition probes first in anagram form (Peynircioğlu \& Tekcan, 1993; Peynircioğlu \& Watkins, 1988; Watkins \& Peynircioğlu, 1990; Westerman \& Greene, 1996), in degraded form (Luo, 1993), or in reverse typing (Luo, 1993), or when participants are required to guess the identity of a gradually revealed recognition probe (Hicks \& Marsh, 1998; LeCompte, 1995; Peynircioğlu \& Tekcan, 1993; Watkins \& Peynircioğlu, 1990). Revelation effects are robust: A meta-analysis conducted by Hicks and Marsh (1998) based on results from 32 experimental conditions showed that revelation effects occurred in all but four of the tasks.

\section{Memory Sensitivity Accounts of the Revelation Effect: Familiarity Change}

Initial accounts of the revelation effect proposed that the revelation task increased the familiarity of the subsequently presented recognition probe, thereby increasing the hit and false alarm rates (LeCompte, 1995; Luo, 1993). There is some evidence to support this notion. Revelation effects are absent for tasks that rely on recollective processes, such as associative recognition and plurality discrimination (Cameron \& Hockley, 2000; Westerman, 2000). Additionally, LeCompte's (1995) results based on both the remember/know and the process dissociation procedures are consistent with the view that the revelation task only influences familiarity-based decisions.

Westerman and Greene (1998) proposed that revelation tasks activate information present in episodic memory that is related to, but not directly activated by, the recognition probe. This additional activation accumulates with the activation that occurs from the test probe, thereby increasing the overall activation and thus the level of familiarity of the test probe. However, the degree of orthographic or categorical similarity between the revelation task stimuli and the recognition probe does not mediate the size of revelation effect (Peynircioğlu \& Tekcan, 1993). In fact, a wide variety of unrelated revelation tasksnumber strings, arithmetic problems, and memory span tests (Bornstein \& Neely, 2001; Niewiadomski \& Hockley, 2001; Verde \& Rotello, 2003; Westerman \& Greene, 1996, 1998) - have produced revelation effects, indicating that revelation effects are not likely mediated by the similarity of the revelation and recognition items. Additionally, revelation effects are similar when revelation tasks involve solving anagrams of either previously studied or unstudied items (Verde \& Rotello, 2003) indicating that memory for the revelation item itself does not influence the revelation effect. Furthermore, revelation effects are not amplified when recognition decisions are preceded by two rather than one revelation task (Niewiadomski \& Hockley, 2001).

\section{Criterion Change Accounts}

Other researchers have proposed that rather than increasing familiarity, the revelation task influences the sub-

W. E. Hockley, whockley@wlu.ca 
jects' setting of their recognition decision criterion. For instance, Hicks and Marsh (1998) suggested that solving the revelation task activates a variety of alternative items in memory, making it harder to discriminate between studied and unstudied recognition probes. Accordingly, subjects relax their response criteria when making recognition judgments following revelation tasks. Niewiadomski and Hockley (2001) proposed that the revelation task displaces the study list context from working memory. This loss increases the difficulty of the recognition task, leading subjects to adopt a more liberal decision criterion for recognition decisions preceded by revelation tasks.

Additional support for criterion change accounts comes from analyses of receiver operating characteristic (ROC) curves. Specifically, in three experiments in which the revelation task item was different from the recognition probe, Verde and Rotello (2003) found a revelation effect but no change in discrimination, leading them to argue in favor of a criterion change interpretation of the revelation effect.

\section{Two Types of Revelation Effects?}

Verde and Rotello (2004) proposed that the principal mechanism that mediates revelation effects is different depending on the nature of the revelation task. When a revelation task involves an item that differs from the recognition probe (different revelation condition), a more liberal decision criterion change is presumed responsible for the revelation effect. In contrast, when a revelation task involves an item that is identical to the recognition probe (same revelation condition), changes in memory sensitivity are assumed to underlie the revelation effect.

In Verde and Rotello's (2004) study, subjects unscrambled words that were either the same or different than the following recognition test probes. Analysis of recognition decisions in the same and different revelation conditions indicated that while hits and false alarms increased at a similar rate following different revelation tasks, false alarms were greater than hits following same revelation tasks. Analysis of ROC curves revealed that there was no change in memory sensitivity in the different revelation condition compared to the intact condition (as also found in Verde \& Rotello, 2003), but there was a reduction in sensitivity in the same revelation condition compared to the intact condition. These findings led Verde and Rotello (2004) to conclude that, whereas criterion changes are sufficient to explain revelation effects in the different condition, a decrease in memory sensitivity is associated with revelation effects in the same revelation condition.

In an attempt to provide converging evidence for multiple causes of the revelation effect, Leynes, Landau, Walker, and Addante (2005) compared event-related potential (ERP) components linked to recognition decisions preceded by same and different revelation tasks. ERPs have been successfully used to distinguish item familiarity between correct old and new recognition decisions; the ERP old/new effect is considered to be a strong index of item familiarity (e.g., Curran, 1999, 2000). Thus, ERPs provide a possible means to examine the role of familiarity in same and different revelation effects. Leynes et al. found ERP evidence for an initial decrease in familiarity in the same revelation condition that was not observed in the different revelation condition consistent with a decrementto-familiarity account of the same revelation effect. The interpretation of these results, however, is complicated by the fact that Leynes et al. observed old/new ERP differences in the intact condition of each experiment, but not in either the same or the different revelation conditions. A further complication is that Azimian-Faridani and Wilding (2004) found that the ERP index of familiarity was lower for both old and new test items in their examination of the different revelation condition. Thus, the current ERP evidence does not provide consistent or conclusive support for different bases of same and different revelation effects.

The present set of experiments was designed to provide converging evidence for Verde and Rotello's (2004) two-factor account of the revelation effect using a twoalternative forced-choice test (2-AFC) procedure. When subjects are forced to choose between two items on a recognition test, they can base their recognition decisions on the relative familiarity of the two alternatives instead of comparing the familiarity of a single test item to an established criterion. Although there is some controversy as to whether forced-choice and yes-no recognition tasks differ with regards to the relative ease and the accuracy of the estimates of discrimination of each procedure, it is generally agreed that forced-choice tests minimize response bias that may occur in yes-no item recognition. ${ }^{1}$ In effect, finding a revelation effect in the forced-choice test procedure would provide strong support for memory sensitivity change explanations of the effect. If no revelation effect is found, however, criterion change explanations of the revelation effect in yes-no recognition would gain support.

Hicks and Marsh (1998) used a 2-AFC recognition test to examine the revelation effect in the same condition; the revelation task always involved one of the two subsequently presented recognition alternatives. A significant "anti-revelation effect" was found where subjects were less likely to call the revealed target item old compared to the other test alternative. This finding is consistent with Verde and Rotello's (2004) finding of a reduction in memory sensitivity in the same revelation condition.

Hicks and Marsh (1998) did not examine the revelation condition that involved items that were different from both recognition alternatives (i.e., the different revelation condition). In the experiments that follow, subjects were administered 2-AFC recognition tests that were presented intact or were preceded by a revelation task that revealed either one of the two recognition alternatives or neither alternative. We hypothesized that if memory sensitivity change underlies the revelation effect in the same revelation condition, a revelation effect should emerge as in Hicks and Marsh (1998). In contrast, a revelation effect should not be seen in the different revelation condition if this revelation effect occurs in yes-no recognition because of a change in decision criterion.

The stimuli were words in Experiment 1 whereas nonwords were tested in Experiment 2. Several studies have found the revelation effect for nonwords in the different revelation condition using the yes-no recognition procedure (Hockley \& Niewiadomski, 2001; Westerman 
\& Greene, 1998; Whittlesea \& Williams, 2001). To our knowledge, the revelation effect has not been examined for nonwords in the same revelation condition.

\section{EXPERIMENTS 1 AND 2}

\section{Method}

Subjects. Twenty-eight students participated in Experiment 1, and 36 students participated in Experiment 2. The students received course credit for participation.

Apparatus and Stimuli. The 140 words and anagrams were chosen from Gibson and Watkins's (1988) pool of eight-letter words. The 128 eight-letter nonwords and nonword anagrams were graphemically regular and pronounceable letter strings (e.g., hillcurt, splookes, lobisner, fomicked, dallower) adapted from Ratcliff, Hockley, and McKoon (1985). All word and nonword anagrams were scrambled in the same order and thus had the same solution code: 54687321 (i.e., forensic would appear as nescirof).

Computers controlled stimuli presentation and recorded test responses. Subjects pressed the " $z$ " or " " key if the alternative on the right or left side of the screen was old. Subjects typed solutions to anagrams before pressing the " $y$ " key to proceed to the recognition test.

Procedure. Subjects were initially exposed to a study list containing 72 words (Experiment 1) or 72 nonwords (Experiment 2) that they were asked to remember for a later memory test. The study items were presented one at a time for $2 \mathrm{sec}$ separated by a blank screen for $0.5 \mathrm{sec}$. The first and last four items of the study list were primacy and recency buffers and were not tested. The recognition test consisted of 64 test pairs each containing one study list item (target) and one new item (lure). Twenty-eight of the test pairs were presented intact, whereas the remaining 36 were preceded by an anagram. Twelve of the anagrams were the same as the target, 12 were the same as the lure, and the remaining 12 anagrams were different from both test pair alternatives and all other items in the experiment. The solution code for the anagrams appeared on the screen beneath each anagram. The left-right order of the target and lure in each test pair and the order of the presentations in the test list were randomly determined for each subject. The test list was subject-paced, with a 1-sec blank interval between each response and the following presentation.

Subjects were given the test instructions concerning anagram solution and recognition responding following the study list. They were instructed to solve the anagrams using the solution code, voice the solution aloud and type in the solution, and then press the " $y$ " key to proceed. For the recognition tests, they were instructed to press the " $z$ " key if the left member of the test pair was the studied word or press the "/" key if the right member was previously studied.

\section{Results and Discussion}

Mean proportions of correct responses for both Experiments 1 and 2 are presented in Table 1. A revelation effect was found in the same but not the different revelation condition in both experiments. Paired-samples $t$ tests showed that the hit rate was significantly lower in the same revelation condition compared to the intact control condition for Experiments $1[t(27)=3.05, p=.005]$ and $2[t(35)=$ $2.67, p=.011]$. That is, as observed by Hicks and Marsh (1998), solving an anagram that was the same as the subsequently presented target made it less likely that subjects would select the target as the old alternative.

Critically, there were no effects of revelation in the different revelation condition. Recognition performance was the same in the intact and different revelation conditions for Experiment $1[t(27)=0.638, p=.529]$ and Experiment $2[t(35)=-0.485, p=.631]$. This finding supports the contention set forth by Verde and Rotello (2004) that
Table 1

Mean Proportion of Correct Responses in the Intact, Different, Same (Distractor), and Same (Target) Revelation Conditions for Experiment 1 (Words) and Experiment 2 (Nonwords)

\begin{tabular}{|c|c|c|c|c|}
\hline \multirow[b]{3}{*}{ Test Condition } & \multicolumn{4}{|c|}{ Proportion Correct } \\
\hline & \multicolumn{2}{|c|}{$\begin{array}{c}\text { Words } \\
\text { (Experiment 1) }\end{array}$} & \multicolumn{2}{|c|}{$\begin{array}{c}\text { Nonwords } \\
\text { (Experiment 2) }\end{array}$} \\
\hline & $M$ & $S E$ & $M$ & $S E$ \\
\hline Intact & .78 & .02 & .71 & .02 \\
\hline Revelation different & .76 & .03 & .73 & .02 \\
\hline Revelation same (distractor) & .77 & .04 & .80 & .02 \\
\hline Revelation same (target) & .70 & .03 & .63 & .03 \\
\hline
\end{tabular}

revelation effects in the different revelation condition of the yes-no recognition procedure are mediated only by a change in the decision criterion.

The results of Experiments 1 and 2 differed in one respect. In Experiment 1, subjects were equally likely to classify a lure as old when it was revealed before the recognition decision or presented intact $[t(27)=0.044, p=.965]$. In contrast, in Experiment 2 there was an effect of revelation for nonword lures $[t(35)=-3.31, p=.002]$, such that subjects were less likely to select the lure when it was revealed in the anagram task (and thus were more likely to chose the target item). Hicks and Marsh (1998) also obtained a similar effect in one of their four experiments.

A third experiment was conducted in which study and test lists contained both word and nonword stimuli in order to compare the revelation effects for these two types of stimuli directly. Hockley and Niewiadomski (2001) found a different condition revelation effect for nonwords in pure but not in mixed lists with words. They argued that this interaction was due to changes in yes-no recognition decision criteria between words and nonwords that negated criterion changes in the revelation condition for nonwords. Since such criterion changes do not play a role in forcedchoice recognition decisions, we expected to see a revelation effect for both words and nonwords in mixed lists in the same but not the different revelation conditions.

\section{EXPERIMENT 3}

\section{Method}

Subjects. Forty-four students participated in exchange for course credit.

Apparatus and Stimuli. The stimuli and apparatus were the same as in Experiments 1 and 2.

Procedure. With the following exceptions, the procedure was identical to that employed in the previous experiments. The study list was composed of a randomly mixed list of 52 words and 52 nonwords. In addition there were two words and two nonwords presented at the beginning and end of the study list to absorb primacy and recency effects. The 2-AFC test list consisted of 52 word pairs and 52 nonword pairs. Each of the 52 test presentations consisted of 13 intact presentations, 13 pairs preceded by an anagram of the target item, 13 preceded by an anagram of the lure, and 13 preceded by an unrelated anagram that was the same stimulus type as the test pair.

\section{Results and Discussion}

The mean proportions of correct responses in each test condition are presented in Table 2. A revelation effect was found in the same but not the different revelation condi- 
tions. Planned paired-samples $t$ tests showed that the hit rates were significantly lower in the same revelation target condition compared to the intact control condition for both words $[t(43)=2.12, p=.04]$ and nonwords $[t(43)=3.58$, $p=.001]$. In contrast, there was no significant difference in performance between the different revelation and intact conditions for either words $[t(43)=-0.39, p=.701]$ or nonwords $[t(43)=1.52, p=.137]$.

In Experiment 1, subjects were equally likely to classify a lure as old when it was revealed before the recognition decision or presented intact, whereas in Experiment 2 subjects were less likely to select the lure when it was revealed in the anagram task. This effect was not replicated in Experiment 3. Accuracy was similar in the same lure revelation condition and the intact condition for words $[t(43)=$ $0.49, p=.627]$ and for nonwords $[t(43)=0.47, p=.638]$. Thus, in contrast to revealing the target item, revealing the incorrect alternative prior to a forced-choice decision does not have a very robust or consistent effect on recognition performance (see also Hicks \& Marsh, 1998).

Experiment 3 replicated the principal finding of Experiments 1 and 2, namely a revelation effect when the revelation task revealed the target item but no revelation effect when the revealed item was unrelated to the test alternatives. The finding of a revelation effect for both words and nonwords in mixed lists for the same but not the different revelation condition is also consistent with Hockley and Niewiadomski's (2001) view that the failure to find a different revelation effect for nonwords in mixed lists of words and nonwords in yes-no recognition is due to competing changes in decision criteria.

\section{GENERAL DISCUSSION}

We examined Verde and Rotello's (2004) hypothesis that there are two types of revelation effects, one associated with changes in memory sensitivity and one mediated by changes in decision criteria. By minimizing the role of response bias through use of a forced choice design, we replicated the findings of Hicks and Marsh (1998) by obtaining a revelation effect for recognition decisions in the same revelation condition. We also extended Hicks and Marsh's results, demonstrating that a revelation effect is not found in the different revelation condition, and that the effects of the same and different revelation conditions are similar for both words and nonwords. These results, together with the ROC analyses reported by Verdes and Rotello (2004), provide converging evidence that while a

Table 2

Mean Proportion of Correct Responses in the Intact, Different, Same (Distractor), and Same (Target) Revelation Conditions of Experiment 3 for Word and Nonword Stimuli

\begin{tabular}{|c|c|c|c|c|}
\hline \multirow[b]{3}{*}{ Test Condition } & \multicolumn{4}{|c|}{ Proportion Correct } \\
\hline & \multicolumn{2}{|c|}{ Words } & \multicolumn{2}{|c|}{ Nonwords } \\
\hline & $M$ & $S E$ & $M$ & $S E$ \\
\hline Intact & .82 & .02 & .76 & .02 \\
\hline Revelation different & .84 & .04 & .72 & .03 \\
\hline Revelation same (distractor) & .81 & .03 & .74 & .03 \\
\hline Revelation same (target) & .77 & .02 & .65 & .03 \\
\hline
\end{tabular}

decrease in sensitivity is involved in the same condition revelation effect, criterion changes drive the different condition revelation effect in tests of yes-no recognition.

It is generally agreed that response bias in the revelation condition occurs because these recognition decisions are perceived or experienced as more difficult compared to recognition decisions presented intact (Hicks \& Marsh, 1998; Hockley \& Niewiadomski, 2001; Leynes, et al., 2005; Verde \& Rotello, 2003). As Hicks and Marsh (1998, p. 1116) discuss, more difficult tests produce more liberal shifts in criterion. Thus, while the task-switching requirements of the revelation procedure in the different condition directly or indirectly give rise to a perception of increased difficulty, they do not result in a decrease in discrimination performance.

Although the same revelation task condition is associated with a decrease in familiarity, it is not clear why solving an anagram of the probe would have such an effect. One possibility suggested by Hicks and Marsh (1998) is that the revelation task activates competing alternatives not activated by the probe alone, and these additional competitors reduce the signal-to-noise ratio of the revealed probe. The smaller signal-to-noise ratio makes the probe less familiar. A problem for this account, however, is that one would expect that a revelation task involving an item (e.g., raindrop) unrelated to the test probe (e.g., vineyard) should activate as many, if not more, competing alternatives than are activated in the course of solving an anagram of vineyard and therefore also function to reduce the signal-to-noise ratio for vineyard. Thus, Hicks and Marsh's familiarity-decrement account requires the additional assumption that the activated competitors be associated in some manner with the test probe. Furthermore, as the revelation effect is similar for nonwords as for words, the relationship between the competitors and the test probe is unlikely to be semantic in nature.

Another possible explanation is that the activation engendered in revealing the probe in an unrelated context serves to interfere with the retrieval of, or reduce access to, the representation of related information in the study list context thereby reducing the familiarity of old and new tests. Such interference would be more detrimental for targets than lures, a finding observed in the present results and in Hicks and Marsh (1998), and also observed in the same condition in yes-no recognition (e.g., Verde \& Rotello, 2004).

A decrease in familiarity, regardless of the reason, provides a reasonably straightforward account of same revelation effects in forced-choice recognition if it is assumed that discrimination in this task is based to a large degree on a relative assessment of familiarity. Decreasing the familiarity of the target item would increase the difficulty of the discrimination and the proportion of correct responses would suffer accordingly. Decreasing the familiarity of the incorrect alternative would have less of an influence on performance.

Participants might also exaggerate the effects of decreases in familiarity of revealed probes in the forced-choice procedure by adopting a discounting strategy (see Jacoby \& Whitehouse, 1989). That is, participants might attribute some of the familiarity associated with the revealed test 
alternative to the revelation task leading them to discount to some degree the familiarity of the revealed probe. This discounting might be done to a greater extent for revealed targets than for revealed lures because the target items would have a higher overall familiarity value than the lures.

A decrease in familiarity by itself, however, does not provide a straightforward account of the same revelation effect in yes-no recognition. If the familiarity of the old and new items in the same revelation condition decreased relative to the familiarity of the old and new items in the intact condition, and participants did not change their decision criterion, then one would expect to see lower hit and false alarm rates in the revelation condition rather than the observed increase. Thus, a decrease in familiarity must also be accompanied by a change in response bias (AzimianFaridani \& Wilding, 2004), or a change in how the attribution of familiarity is made between the revelation task and study list (see Whittlesea \& Williams, 2001).

In summary, the present results based on the forcedchoice procedure complement recent findings using yesno recognition to support Verde and Rotello's (2004) twofactor account of the revelation effect. In this view, when the revealed item is the same as the target item the revelation effect is associated with a decrease in memory sensitivity whereas when the revealed item is unrelated to the target the revelation effect is due to a change in decision bias.

\section{AUTHOR NOTE}

This study was supported by a discovery grant from the National Science and Engineering Research Council of Canada to the second author. We thank the anonymous reviewers for their very constructive comments on earlier drafts of the manuscript. Correspondence can be addressed to either J. C. Major or W. E. Hockley, Department of Psychology, Wilfrid Laurier University, Waterloo, ON, N2L 3C5 Canada (e-mail: majo1929@wlu.ca; whockley@wlu.ca).

\section{REFERENCES}

Azimian-Faridani, N., \& Wilding, E. L. (2004). An event-related potential study of the revelation effect. Psychonomic Bulletin \& Review, 11, 926-931.

Bornstein, B. H., \& Neely, C. B. (2001). The revelation effect in frequency judgment. Memory \& Cognition, 29, 209-213.

Cameron, T. E., \& Hockley, W. E. (2000). The revelation effect for item and associative recognition: Familiarity versus recollection. Memory \& Cognition, 28, 176-183.

Curran, T. (1999). The electrophysiology of incidental and intentional retrieval: ERP old/new effects in lexical decision and recognition memory. Neuropsychologia, 37, 771-785.

Curran, T. (2000). Brain potentials of recollection and familiarity. Memory \& Cognition, 28, 923-938.

Gibson, J. M., \& WatKins, M. J. (1988). A pool of 1,086 words with unique two-letter fragments. Behavior Research Methods, Instruments, \& Computers, 20, 390-397.

HiCKS, J. L., \& MARSH, R. L. (1998). A decrement-to-familiarity interpretation of the revelation effect from forced-choice tests of recognition memory. Journal of Experimental Psychology: Learning, Memory, \& Cognition, 24, 1105-1120.

Hockley, W. E., \& NieWIAdOMSKI, M. W. (2001). Interrupting recognition memory: Tests of a criterion-change account of the revelation effect. Memory \& Cognition, 29, 1176-1184.

JACOBY, L. L., \& Whitehouse, K. (1989). An illusion of memory: False recognition influenced by unconscious perception. Journal of Experimental Psychology: General, 118, 126-135.

Kroll, N. E. A., Yonelinas, A. P., Dobbins, I. G., \& Frederick, C. M. (2002). Separating sensitivity from response bias: Implications of comparisons of yes-no and forced-choice tests for models and measures of recognition memory. Journal of Experimental Psychology: General, 131, 242-254.

LeCompte, D. C. (1995). Recollective experience in the revelation effect: Separating the contribution of recollection and familiarity. Memory \& Cognition, 23, 324-334.

Leynes, P. A., Landau, J., Walker, J., \& Addante, R. J. (2005). Event-related potential evidence for multiple causes of the revelation effect. Consciousness \& Cognition, 14, 327-350.

LuO, C. R. (1993). Enhanced feeling of recognition: Effects of identifying and manipulating test items on recognition memory. Journal of Experimental Psychology: Learning, Memory, \& Cognition, 13, 405-413.

Macmillan, N. A., \& Creelman, C. D. (1991). Detection theory: A user's guide. New York: Cambridge University Press.

Niewiadomski, M. W., \& HockLeY, W. E. (2001). Interrupting recognition memory: Tests of familiarity-based accounts of the revelation effect. Memory \& Cognition, 29, 1130-1138.

PeynircioĞLu, Z. F., \& TeKCAN, A. (1993). Revelation effect: Effort of priming does not cause the sense of familiarity. Journal of Experimental Psychology: Learning, Memory, \& Cognition, 19, 382-388.

Peynircioğlu, Z. F., \& Watkins, M. J. (1988). Effect of unfolding stimulus presentation on recognition memory. In M. M. Gruneberg, P. E. Morris, \& R. Sykes (Eds.), Practical aspects of memory: Current research and issues (Vol. 2, pp. 518-523). Chichester, U.K.: Wiley.

RatCliff, R., Hockley, W. E., \& McKoon, G. (1985). Components of activation: Repetition and priming effects in lexical decision and recognition. Journal of Experimental Psychology: General, 114, 435-450.

Verde, M. F., \& Rotello, C. M. (2003). Does familiarity change in the revelation effect? Journal of Experimental Psychology: Learning, Memory, \& Cognition, 29, 739-746.

Verde, M. F., \& Rotello, C. M. (2004). ROC curves show that the revelation effect is not a single phenomenon. Psychonomic Bulletin \& Review, 11, 560-566.

Watkins, M. J., \& PeynircioĞLu, Z. F. (1990). The revelation effect: When disguising test items induces recognition. Journal of Experimental Psychology: Learning, Memory, \& Cognition, 16, 1012-1020.

Westerman, D. L. (2000). Recollection-based recognition eliminates the revelation effect in memory. Memory \& Cognition, 28, 167-175.

Westerman, D. L., \& Greene, R. L. (1996). On the generality of the revelation effect. Journal of Experimental Psychology: Learning, Memory, \& Cognition, 22, 1147-1153.

Westerman, D. L., \& Greene, R. L. (1998). The revelation that the revelation effect is not due to revelation. Journal of Experimental Psychology: Learning, Memory, \& Cognition, 24, 377-386.

Whittlesea, B. W. A., \& Williams, L. D. (2001). The discrepancyattribution hypothesis: I. The heuristic basis of feelings and familiarity. Journal of Experimental Psychology: Learning, Memory, \& Cognition, 27, 3-13.

\section{NOTE}

1. Forced-choice performance can be influenced by a participant's bias to choose the left or right test alternative more often than the other (Macmillan \& Creelman, 1991). Such a position bias would serve to attenuate differences between conditions when the correct alternative is equally likely in each position, and is a very different type of bias than the conservative or liberal response biases that can occur in the yesno recognition procedure. See Kroll, Yonelinas, Dobbins, and Frederick (2002) for a recent comparison of forced-choice and yes-no recognition test procedures.

(Manuscript received July 28, 2006; revision accepted for publication April 23, 2007.) 